Article

\title{
Sport-Specific Differences in Power-Velocity-Force Profiling during Trunk Rotations at Different Loads
}

\author{
Erika Zemková $^{1,2, *}$, Oliver Poór ${ }^{1}$ and Michal Jeleň ${ }^{2}$ (i) \\ 1 Faculty of Physical Education and Sports, Comenius University in Bratislava, 81469 Bratislava, Slovakia; \\ oliverpoor55@gmail.com \\ 2 Sports Technology Institute, Faculty of Electrical Engineering and Information Technology, \\ Slovak University of Technology, 81219 Bratislava, Slovakia; michal.jelen@stuba.sk \\ * Correspondence: erika.zemkova@uniba.sk; Tel.: +421-2-206-69-951
}

Received: 29 October 2020; Accepted: 22 November 2020; Published: 25 November 2020

Featured Application: While force-velocity-power characteristics of resistance exercises, such as squats and bench presses, have been well documented, little attention has been paid to load, force and power-velocity relationships in trunk rotation exercises. This study revealed that the highest power is produced by fighting sports athletes, followed by those of water, combat and ball sports, with the maximum achieved at 10.5, 20.0, 15.5 and $10.5 \mathrm{~kg}$, respectively. Additionally, angular velocity is the highest at lower weights in fighting sports athletes and at higher weights in water sports athletes. Alternatively, the highest force is achieved at higher velocities in fighting sports athletes and at lower velocities in water sports athletes. These findings indicate that power, velocity and force produced during trunk rotations are sensitive parameters able to discriminate between athletes with different demands on explosive strength and power of core muscles.

\begin{abstract}
This study investigates differences in power and velocity at different loads and power and force at different velocities during trunk rotations in athletes who practice sports with rotational demands on the trunk. Athletes of combat $(n=23)$, fighting $(n=39)$, ball $(n=52)$ and water sports $(\mathrm{n}=19)$ with a mean age of $23.8 \pm 1.5$ years performed standing trunk rotations on each side with bars of different weights (from $1 \mathrm{~kg}$ up to $50 \mathrm{~kg}$ ) placed on their shoulders. The findings showed significant between-group differences in mean power in the acceleration phase of trunk rotations, especially at higher weights $(\geq 10.5 \mathrm{~kg})$ or lower velocities $(\leq 334.2 \mathrm{rad} / \mathrm{s})$. The power at $10.5 \mathrm{~kg}$ was significantly higher in fighting than water $(p=0.035 ; \mathrm{d}=0.86)$, combat $(p=0.001 ; \mathrm{d}=1.53)$ and ball sports athletes $(p=0.001 ; \mathrm{d}=1.48)$, with no significant differences between the two latter groups; at $15.5 \mathrm{~kg}$, it was higher in water than combat $(p=0.027 ; \mathrm{d}=0.91)$ and ball sports athletes $(p=0.009 ; \mathrm{d}=1.17)$ but not those in fighting sports; and at $20 \mathrm{~kg}$, it was higher in water than combat $(p=0.013 ; \mathrm{d}=0.98)$ and ball sports athletes $(p=0.006 ; \mathrm{d}=1.33)$, with no significant differences with those in fighting sports. This testing is sensitive in discriminating between athletes of various sports, which may reflect the specificity of their training, including trunk rotations at various velocities under different load conditions.
\end{abstract}

Keywords: angular velocity; athletes; muscle power; rotational movement of the trunk

\section{Introduction}

Exercises for strengthening core muscles are currently being widely promoted. These exercises have been used as a part of prevention and rehabilitation programs for musculoskeletal injuries $[1,2]$. However, limited and conflicting evidence exists regarding their efficiency for the improvement of sporting performance. This is most likely due to the lack of sport-specific tests evaluating the stability 
and strength of core muscles. Sport training induces specific adaptations of the core, which may not be revealed using non-specific tests [3]. In practice, the biomechanical analysis of techniques, the experience of strength and conditioning specialists and their training evidence have been generally used.

Another drawback is that most of the tests do not target the spine-stabilizing muscles despite the fact that the most important stabilizers are task specific [4]. Selecting an appropriate test to assess core muscle stability and strength is difficult because of the interaction of the lumbopelvic hip structures. Core stability in static single-joint and dynamic multi-joint exercises may not correlate due to its high level of task specificity [5]. Static core muscle stability and strength tests may therefore be unable to distinguish between power-trained athletes and assess the effect of training involving dynamic core muscle exercises. This low sensitivity of current testing methods limits their practical applications.

Tests used to assess active trunk control during functional tasks are restricted to linear movements that lack the explosive movements associated with sporting activities [6]. In practice, a medicine ball or cable pulleys can be used for this purpose, as they allow motion in all three planes. For instance, one can use a device that allows the monitoring of biomechanical variables during trunk rotations in the form of a modified woodchop exercise while sitting [7] or standing [8]. The protocol including a diagonal chop and lift power exercise generates reliable data and it seems to simulate dynamic movement of the trunk [6]. Although rotational power is a fundamental component in functional tasks, such as striking or throwing, Talukdar et al. [9] suggest that rotational power measured during the chop and lift may not be an essential factor in throwing velocity in cricket.

The external validity of isometric and isokinetic strength tests of the trunk muscles for functional tasks is also ambiguous. Some authors have documented the relationship between measures of core muscle strength and athlete performance [10,11], whereas others have not [12-14]. The relationship between the limbs and core muscles has been demonstrated for various lifting tasks, forehand and backhand strokes in tennis, overhead throwing in baseball and so forth [15-21]. These findings highlight the importance of core musculature in the transfer of momentum and torque throughout the kinetic chain during sport-specific tasks [4].

Furthermore, there are no significant differences in peak torque measured using an isokinetic dynamometer between and within groups of highly skilled golfers and healthy individuals who do not play golf [22]. Further, no significant differences were found in the trunk muscle endurance between the elite golfers and the controls [22]. Similarly, no significant differences in the static holding times and a decline in the electromyography (EMG) median frequency between low-handicap golfers with low back pain and healthy non-golfing controls were reported [23]. The shortcomings of these laboratory tests is that strength characteristics of core muscles are measured when sitting in a fixed position. However, core muscles facilitate trunk movements more easily when the body is in an upright position [24], thus between- and within-group differences in strength and power produced during trunk rotations can be better revealed.

Therefore, to be relevant to a particular sport, core muscle stability and strength tests must mimic their specific movements very closely. However, none have truly evaluated core stability and core strength under sport-specific conditions, which are far removed from laboratory tests. Therefore, further research is needed to elucidate whether between- and within-group differences in trunk muscle power exist in athletes with different demands on velocity and power production during trunk rotations.

This study investigates differences in power and velocity at different loads, and power and force at different velocities during trunk rotations in athletes of combat sports (judo, wrestling), fighting sports (boxing, Thai boxing, karate, tae kwon do), ball sports (golf, hockey, tennis) and water sports (canoeing, kayaking). We hypothesized that fighting sports athletes who have to generate high force outputs in a short amount of time and also ball sports players would produce the highest power at lower weights or at higher velocities, whereas water sports athletes who exert a high force against the water and those of combat sports who require high explosive strength of both the lower and upper body musculature to lift and throw the opponent would be able to produce the highest power at higher weights. 


\section{Materials and Methods}

\subsection{Participants}

Altogether, 133 male combat sports (judo, wrestling), fighting sports (boxing, Thai boxing, karate, tae kwon do), ball sports (golf, hockey, tennis), and water sports athletes (canoeing, kayaking) volunteered to participate in the study (Table 1). The athletes had over 8 years' experience in their particular sport, including at least 5 years' participation in competition. They also had experience with resistance training involving exercises strengthening the trunk muscles. Athletes who met the inclusion criteria were allocated to four groups. Approximately $83 \%$ of athletes enrolled in sports clubs participated in this study. Athletes were included in this investigation only if they did not subjectively report back pain. Those who had previously undergone medically invasive procedures for the back were excluded. All participants were informed of the main purpose and procedures of this investigation. The procedures followed were in accordance with the ethical standards on human experimentation stated in compliance with the 1964 Helsinki Declaration and its later amendments. Projects were approved by the ethics committee of the Faculty of Physical Education and Sports, Comenius University in Bratislava (Nos. 3/2017, 4/2017 and 1/2020).

Table 1. Participant's characteristics (mean \pm SD).

\begin{tabular}{lcccc}
\hline \multicolumn{1}{c}{ Groups of Athletes } & n (1) & Age (Years) & Height (cm) & Body Mass (kg) \\
\hline Boxers and Thai boxers & 12 & $22.3 \pm 4.7$ & $183.6 \pm 8.5$ & $83.8 \pm 10.0$ \\
Canoeists and kayakers & 19 & $24.7 \pm 3.6$ & $178.6 \pm 6.5$ & $72.7 \pm 6.4$ \\
Golfers & 15 & $26.1 \pm 4.2$ & $180.6 \pm 9.3$ & $81.4 \pm 13.6$ \\
Hockey players & 20 & $23.5 \pm 3.0$ & $184.6 \pm 7.7$ & $83.1 \pm 9.9$ \\
Judo competitors & 14 & $21.9 \pm 2.7$ & $177.3 \pm 6.8$ & $75.6 \pm 7.5$ \\
Karate and tae kwon do competitors & 27 & $22.9 \pm 2.8$ & $179.1 \pm 6.0$ & $76.3 \pm 8.2$ \\
Tennis players & 17 & $23.2 \pm 3.3$ & $181.4 \pm 6.5$ & $73.9 \pm 10.7$ \\
Wrestlers & 9 & $25.4 \pm 3.6$ & $177.9 \pm 8.1$ & $80.0 \pm 8.9$ \\
\hline
\end{tabular}

\subsection{Experimental Protocol}

Participants were asked to avoid high intense exercise preceding and during the study. Prior to testing, they were given a demonstration of the proper technique of trunk rotations. The time of day and testing procedures were identical for all participants. The same researchers conducted the testing in all groups. After a warm-up, participants underwent familiarization trials, during which they performed standing rotations of the trunk in a slow and controlled manner while keeping the back straight. Then they performed two repetitions of trunk rotations to each side per load. Bars of different weights were placed on their shoulders behind the neck. The load increased from $1 \mathrm{~kg}$ by $\sim 5 \mathrm{~kg}$ up to a max. of $50 \mathrm{~kg}$. They were asked to perform rotations of the trunk with maximal effort in the acceleration phase. Emphasis was placed on the correct body position during trunk rotations while standing. Participants stood with their feet wider than shoulder width apart and toes slightly pointed outwards while holding the bar on the shoulders with their hands. They rotated their torso from the right (or the left) side towards the opposite side, and then they slowly returned to the starting position. The test was then repeated for the opposite side of the body. They had to engage their core muscles to stiffen the torso and stabilize the spine. A laboratory assistant ensured that participants remained upright throughout the movement and that their head, chest and torso were aligned over the hips.

During rotational movement of the trunk with a bar over the shoulders, torque has to be produced to accelerate or decelerate mass on both sides of the bar axis. At the same time, centrifugal forces arise. As long as the weights on both sides of the bar, as well as their rotation radii, remain the same, arising centrifugal forces will be of the same magnitude, although acting in opposite directions. This means they will be fully compensated without putting any stress on the trunk of the subject. In order to quantify the torque produced to accelerate (or decelerate) a rotating bar, one needs to 
know the mass of the bar and its rotation radius (distance between the center of gravity of rotating masses and the axis of rotation) and instant acceleration. Calculation of the center of gravity of the total rotating masses on both sides (consisting of the bar axis and weight plates) is based on their geometrical dimensions, assuming the same specific mass of the material (iron) used for the bar axis and weight plates. As the rotation radius and masses are set prior to the test, acceleration depends on the subject's effort. For registration of acceleration, the FiTRO Torso Premium (FiTRONiC, Bratislava, Slovakia) was applied. The equipment consists of an inertia measurement unit (a combination of a three-axis accelerometer and three-axis gyroscope) in a small box with an integrated USB interface connected to the computer. While inserted in the bar axis, the inertia measurement unit registers instant angular velocity of rotation movement (rad per second), which is sampled by the computer at a rate of $100 \mathrm{~Hz}$. Special software calculates instant angular acceleration as a derivation of angular velocity. Radius of rotation (the distance between the center of gravity and the axis of rotation) was used for the transformation of angular velocity (rad per second) and angular acceleration (rad per second squared) into real velocity in meters per second and real acceleration in meters per second squared. Torque produced to accelerate or decelerate rotation movement is calculated by multiplying the moment of inertia and the angular acceleration. This is determined by Newton's second law of motion where the force is equated to the product of the mass times the acceleration. Then the power is calculated as the product of force times velocity.

\subsection{Statistical Analysis}

The statistical program SPSS for Windows version 18.0 (SPSS, Inc., Chicago, IL, USA) was used for data analysis. Data are presented as mean \pm standard deviation (SD). The sample size calculation was conducted with $\alpha=0.05$ ( $5 \%$ chance of type I error) and $1-\beta=0.80$ (power $80 \%$ ) and using the previous results that showed variations in mean power during trunk rotations between athletes of different sports $[25,26]$. This indicated a sample size of 14 per group. Although the sample size in two groups was below this limit, because the inclusion criteria required athletes to be active in their sport, groups of combat sports (judo, wrestling), fighting sports (boxing, Thai boxing, karate, tae kwon do), ball sports (golf, hockey, tennis) and water sports athletes (canoeing, kayaking) were sufficient for finding statistically significant differences. Two-way analysis of variance (ANOVA) was used to determine between-group differences in force, velocity and power during standing trunk rotations with stepwise increasing weights, followed with Bonferroni post hoc tests. The level of significance was set at $p<0.05$. Between-group effect sizes (Cohen's d) were calculated by using a pooled standard deviation. An effect size of 0.80 and higher was considered as large, $0.50-0.79$ as medium, $0.20-0.49$ as small and $0-0.19$ as trivial [27].

\section{Results}

The ANOVA revealed significant between-group differences in power outputs during standing trunk rotations across different loads. As shown in Table 2, the highest values of power were produced by boxers and Thai boxers $(445.3 \pm 43.2 \mathrm{~W}$ at $10.5 \mathrm{~kg})$, followed by hockey players $(407.0 \pm 41.5 \mathrm{~W}$ at $15.5 \mathrm{~kg})$, karate and tae kwon do competitors ( $348.1 \pm 35.4 \mathrm{~W}$ at $10.5 \mathrm{~kg})$, canoeists and kayakers $(346.9 \pm 36.1 \mathrm{~W}$ at $20 \mathrm{~kg})$, wrestlers $(288.7 \pm 27.9 \mathrm{~W}$ at $20 \mathrm{~kg})$, judo competitors $(266.4 \pm 26.3 \mathrm{~W}$ at $15.5 \mathrm{~kg})$, tennis players $(227.3 \pm 22.6 \mathrm{~W}$ at $10.5 \mathrm{~kg})$ and golfers $(169.9 \pm 19.5 \mathrm{~W}$ at $10.5 \mathrm{~kg})$.

When comparing maximal values of power at $10.5 \mathrm{~kg}$, fighting sports athletes were better than water sports $(p=0.035 ; \mathrm{d}=0.86)$, combat sports $(p=0.001 ; \mathrm{d}=1.53)$ and ball sports athletes $(p=0.001$; $\mathrm{d}=1.48$ ), with no significant differences between combat and ball sports athletes (Figure 1). At $15.5 \mathrm{~kg}$, significantly higher values of power were found in water sports athletes than those of combat sports $(p=0.027 ; d=0.91)$ and ball sports $(p=0.009 ; d=1.17)$. However, values did not differ significantly from those achieved by fighting sports athletes. Additionally, at $20 \mathrm{~kg}$, the power was significantly higher in water sports athletes than that of combat sports $(p=0.013 ; \mathrm{d}=0.98)$ and ball sports $(p=0.006$; $\mathrm{d}=1.33$ ) athletes, with no significant differences with fighting sports athletes. 
Table 2. Mean power in the acceleration phase of trunk rotations at different weights in groups of athletes of various sports (mean $\pm \mathrm{SD}$ ).

\begin{tabular}{|c|c|c|c|c|c|c|}
\hline \multirow[b]{2}{*}{ Groups of Athletes } & \multicolumn{6}{|c|}{ Mean Power in the Acceleration Phase of Trunk Rotations at Different Weights (W) } \\
\hline & $1 \mathrm{~kg}$ & $5.5 \mathrm{~kg}$ & $10.5 \mathrm{~kg}$ & $15.5 \mathrm{~kg}$ & $20 \mathrm{~kg}$ & \\
\hline Boxers and Thai boxers & $130.1 \pm 17.8$ & $276.2 \pm 30.1$ & $445.3 \pm 43.2$ & $436.4 \pm 41.8$ & $423.5 \pm 44.1$ & \\
\hline Canoeists and kayakers & $75.1 \pm 9.2$ & $201.3 \pm 20.9$ & $332.6 \pm 25.6$ & $345.3 \pm 30.4$ & $346.9 \pm 36.1$ & \\
\hline Golfers & $60.4 \pm 8.4$ & $118.6 \pm 13.2$ & $169.9 \pm 19.5$ & $136.2 \pm 16.6$ & $107.3 \pm 14.2$ & \\
\hline Hockey players & $120.7 \pm 14.5$ & $236.3 \pm 27.6$ & $393.7 \pm 34.7$ & $407.0 \pm 41.5$ & $404.0 \pm 38.1$ & \\
\hline Judo competitors & $71.0 \pm 9.0$ & $160.7 \pm 19.3$ & $254.2 \pm 25.2$ & $266.4 \pm 26.3$ & $252.3 \pm 27.0$ & \\
\hline Karate and tae kwon do competitors & $99.0 \pm 11.9$ & $213.5 \pm 22.8$ & $348.1 \pm 35.4$ & $321.3 \pm 29.9$ & $271.5 \pm 26.8$ & \\
\hline Tennis players & $95.7 \pm 10.1$ & $173.9 \pm 20.0$ & $227.3 \pm 22.6$ & $213.2 \pm 23.7$ & $183.9 \pm 21.9$ & \\
\hline \multirow[t]{2}{*}{ Wrestlers } & $68.8 \pm 8.9$ & $158.5 \pm 18.9$ & $271.7 \pm 28.5$ & $286.1 \pm 30.3$ & $288.7 \pm 27.9$ & \\
\hline & \multicolumn{6}{|c|}{ Mean Power in the Acceleration Phase of Trunk Rotations at Different Weights (W) } \\
\hline Groups of Athletes & $25 \mathrm{~kg}$ & $30 \mathrm{~kg}$ & $35 \mathrm{~kg}$ & $40 \mathrm{~kg}$ & $45 \mathrm{~kg}$ & $50 \mathrm{~kg}$ \\
\hline Boxers and Thai boxers & $398.8 \pm 35.7$ & $371.0 \pm 33.4$ & $350.9 \pm 34.1$ & $327.3 \pm 29.8$ & $290.1 \pm 26.3$ & $250.5 \pm 25.0$ \\
\hline Canoeists and kayakers & $312.7 \pm 28.0$ & $248.3 \pm 24.7$ & $201.7 \pm 23.4$ & $155.0 \pm 18.1$ & & \\
\hline Golfers & $94.2 \pm 11.1$ & $85.6 \pm 10.5$ & & & & \\
\hline Hockey players & $365.6 \pm 33.2$ & $340.8 \pm 30.9$ & $332.8 \pm 31.9$ & $310.0 \pm 27.7$ & $270.0 \pm 26.9$ & \\
\hline Judo competitors & $214.4 \pm 24.9$ & $180.0 \pm 20.9$ & $155.0 \pm 17.3$ & $110.0 \pm 13.1$ & & \\
\hline Karate and tae kwon do competitors & $232.3 \pm 22.7$ & $200.3 \pm 20.5$ & $165.0 \pm 18.8$ & & & \\
\hline Tennis players & $149.9 \pm 18.4$ & $138.6 \pm 16.3$ & $109.1 \pm 15.0$ & & & \\
\hline Wrestlers & $263.1 \pm 27.4$ & $227.7 \pm 26.6$ & $190.5 \pm 23.5$ & $140.0 \pm 19.9$ & & \\
\hline
\end{tabular}

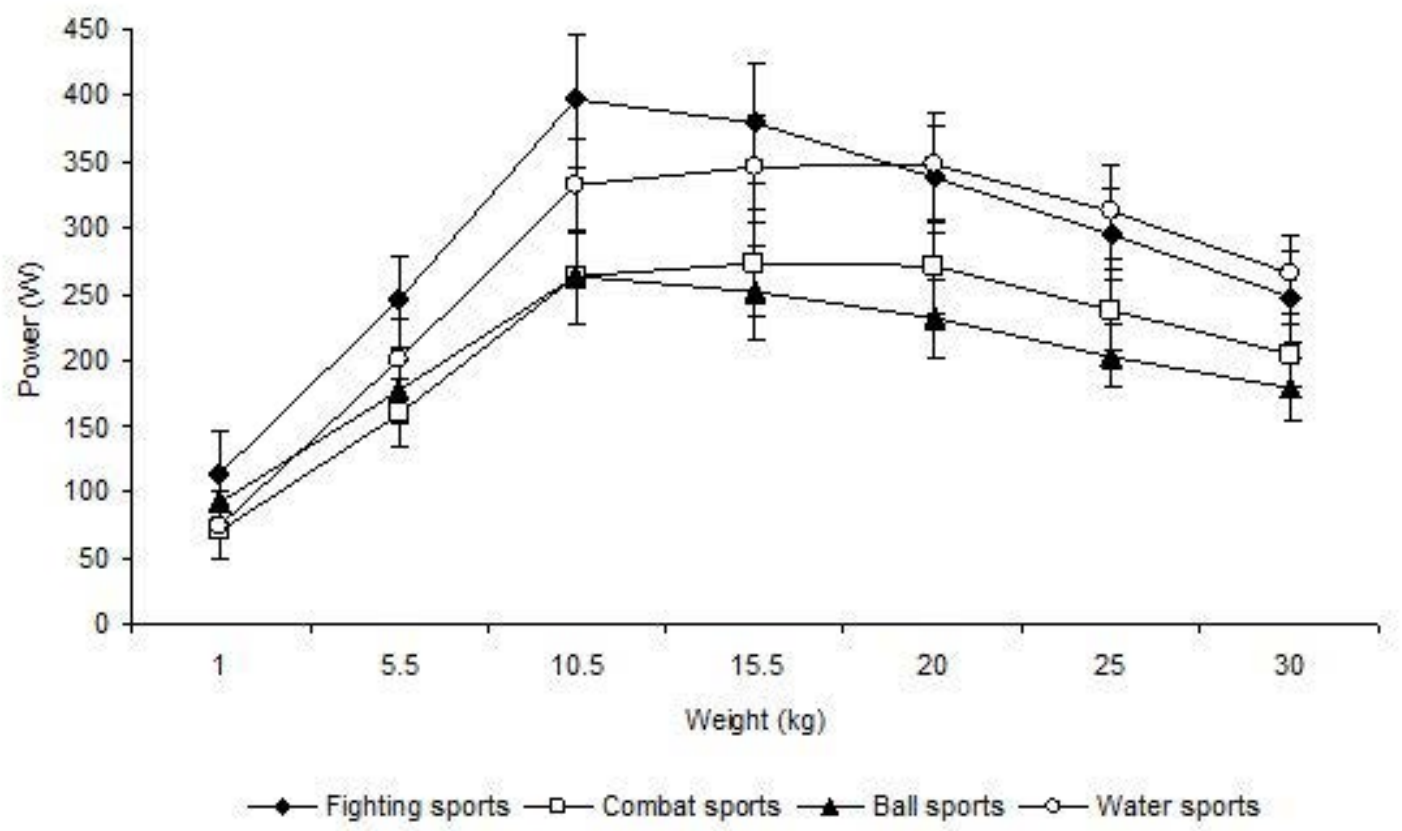

Figure 1. Power during trunk rotations across different weights in ball, combat, fighting and water sports athletes.

Angular velocity showed a similar tendency, depending on the weight used (Figure 2). Interestingly, fighting sports athletes produced greater velocity at lower weights than the remaining three groups of athletes, whereas water sports athletes performed better at higher weights. 


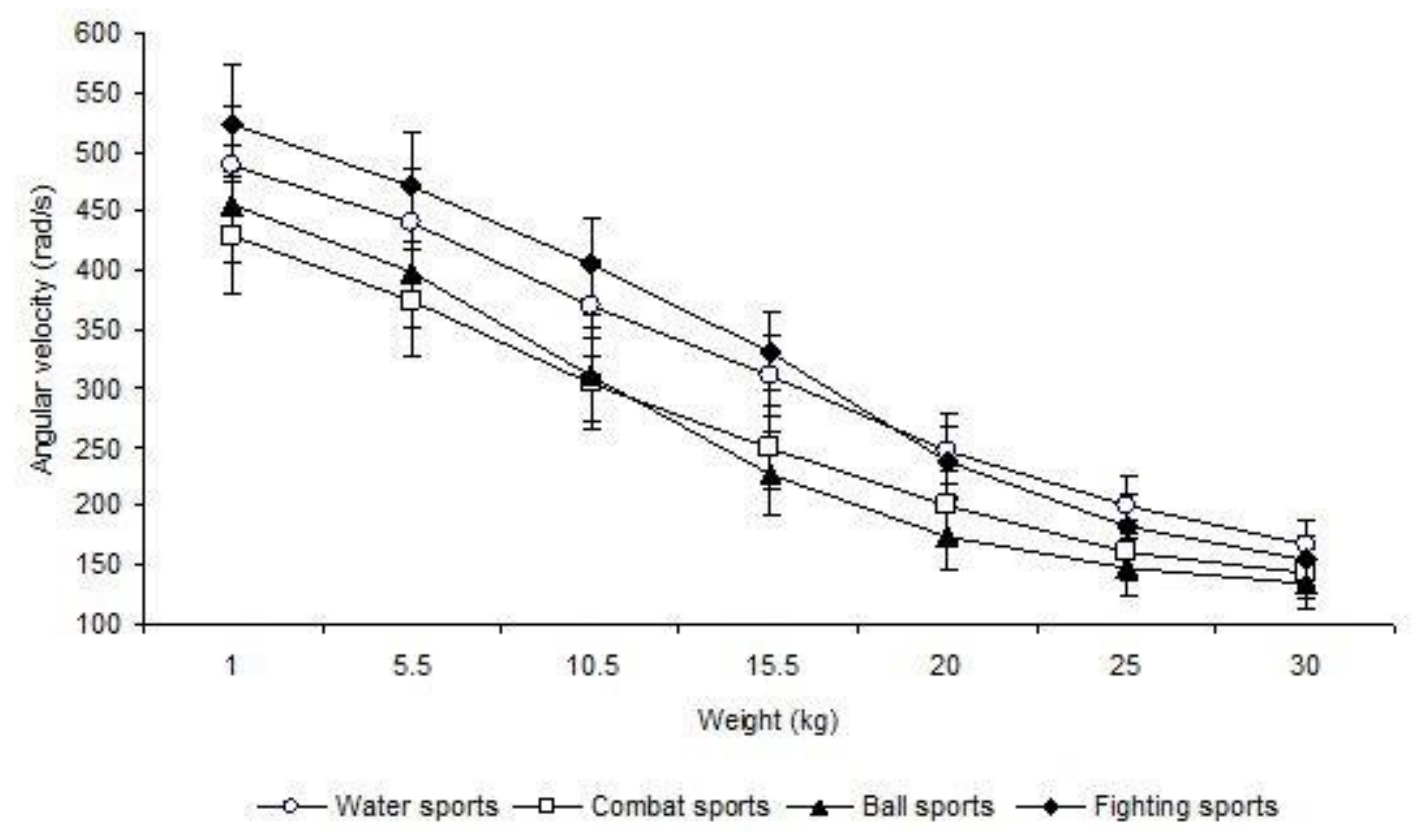

Figure 2. Angular velocity during trunk rotations across different weights in ball, combat, fighting and water sports athletes.

Between-group comparisons also revealed significant differences in power achieved at velocities $\leq 334.2 \mathrm{rad} / \mathrm{s}$ (Figure 3), with the best values identified in fighting sports athletes, followed by those of water sports, combat sports and finally ball sports. While water sports athletes achieved the highest force at lower velocities, fighting sports athletes produced the highest force at higher velocities (Figure 4).

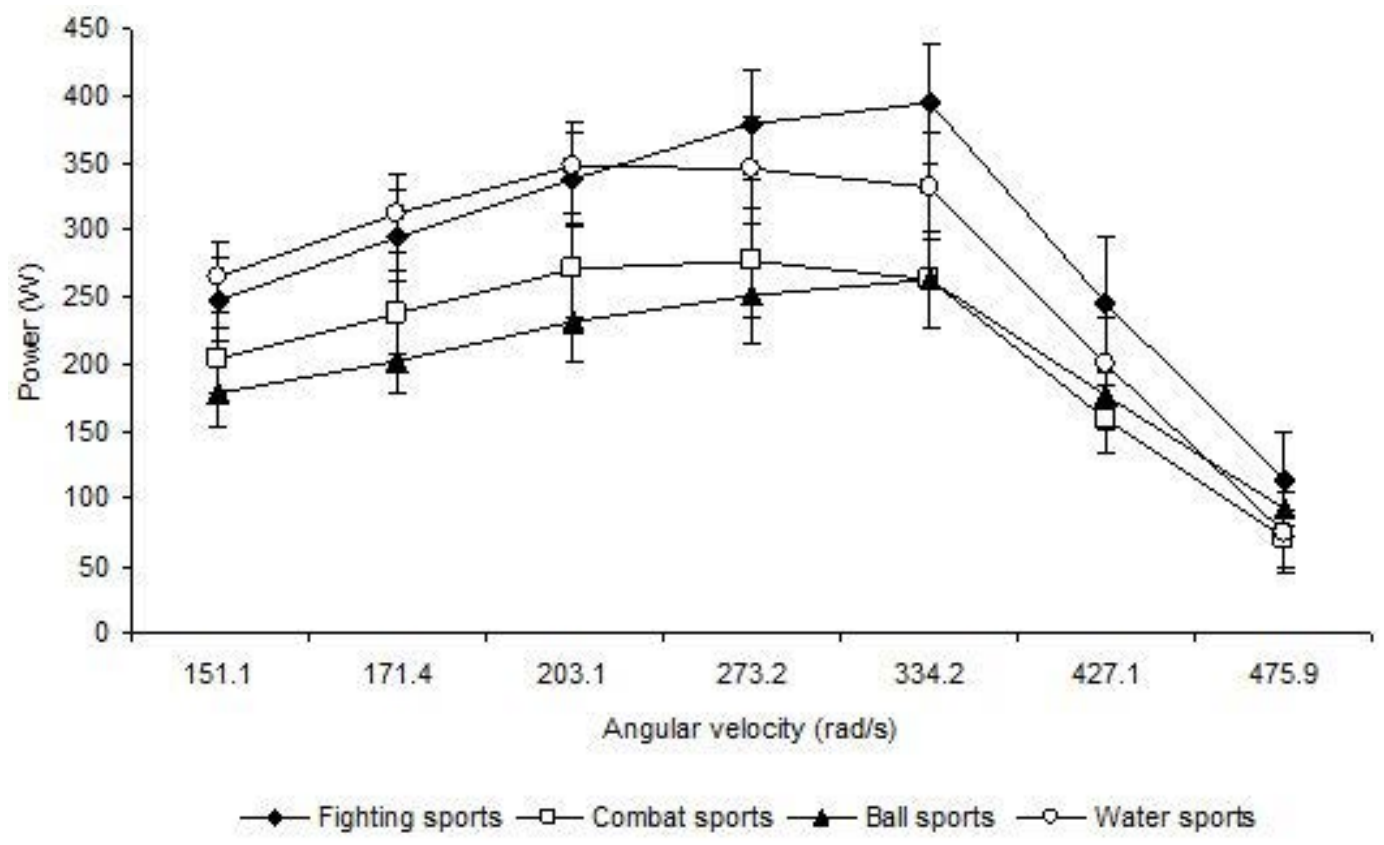

Figure 3. Power during trunk rotations across different velocities in ball, combat, fighting and water sports athletes. 


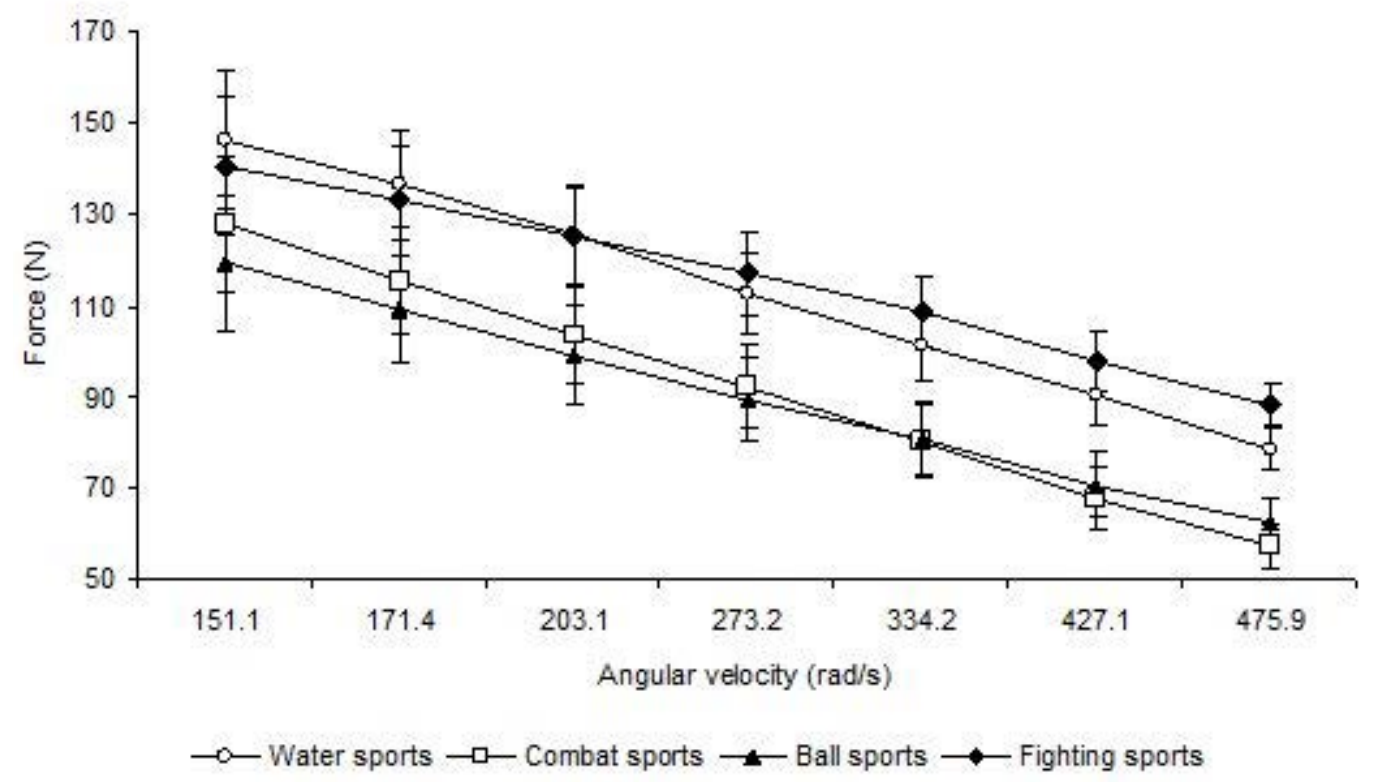

Figure 4. Force during trunk rotations across different velocities in ball, combat, fighting and water sports athletes.

\section{Discussion}

Strength of the trunk muscles contributes significantly to an athlete's ability to transfer forces to the extremities [28]. For instance, the execution of a golf swing or the stroke in tennis or baseball requires rotational power of the trunk, as well as rapid movements of the extremities. Among trunk muscles that are active during the acceleration phase of the golf swing, the highest level of activity was observed in the trail-side abdominal oblique muscles [29]. Preliminary measurements revealed that mean power and velocity in the acceleration phase of seated trunk rotations with 1 and $20 \mathrm{~kg}$ are sensitive variables able to discriminate between- and within-group differences $[25,26]$.

This study identified significant between-group differences in mean power in the acceleration phase of trunk rotations, especially at higher weights $(\geq 10.5 \mathrm{~kg})$ or lower velocities $(\leq 334.2 \mathrm{rad} / \mathrm{s})$. The highest values of power were produced by fighting sports athletes, followed by those of water sports, combat sports and ball sports. Between-group comparisons also revealed that fighting sports athletes achieved the highest power of the three remaining groups at $10.5 \mathrm{~kg}$; however, values at a higher weight of $20 \mathrm{~kg}$ were similar to those produced by water sports athletes. Furthermore, ball sports athletes achieved higher values of power at lower weights (or at higher velocities) than combat and water sports athletes, but the latter two groups performed better at higher weights. Similarly, angular velocity at lower weights was the highest in fighting sports athletes but water sports athletes produced higher velocity at higher weights. In spite of the lower force at lower velocities in athletes of fighting as opposed to water sports, they were able to produce higher force at higher velocities. These between-group differences in the velocity and power produced during trunk rotations may be ascribed to specificity of their training involving movements of the trunk at different velocities under various load conditions. While ball and fighting sports athletes who have to generate high force outputs in a short amount of time achieved the highest power at lower weights or at higher velocities, water sports athletes who exert a high force against the water and those of combat sports who require high explosive strength of both the lower and upper body musculature to lift and throw the opponent were able to produce the highest power at higher weights.

Indeed, the training of canoeists was shown to significantly increase mean power in the acceleration phase of trunk rotations with weights $\geq 10 \mathrm{~kg}$ after the preparatory period, whereas no significant changes after the competitive period were observed [30]. Similarly, values significantly increased in hockey players during trunk rotations with weights $\geq 12 \mathrm{~kg}$ only after the preparatory period [30]. 
However, there was a significant increase in mean power in tennis players after the preparatory (at weights from 10 to $26 \mathrm{~kg}$ ) as well as the competitive period (at weights from 6 to $26 \mathrm{~kg}$ ) [30]. This indicates that changes in power produced during trunk rotations reflect their sport-specific training.

Therefore, testing conditions should be close to those used during competition and training. Commonly, athletes perform resistance exercises with increasing weights up to one repetition maximum (1RM). Then individual power-velocity and force-velocity curves can be provided. In addition, the relationship between power or velocity and the load can be analyzed. While the velocity in the concentric phase of lifting decreases with increasing weights, the power increases from lower weights and, after reaching a peak, decreases again toward higher weights. The optimal velocity at which the highest power is produced depends on the ratio of slow and fast twitch muscle fibers [31] and, therefore, it may be hardly changed with training. However, the optimal weight at which maximal values of power are achieved may increase with training. In the present study, most athletes achieved maximal values of power at $10.5 \mathrm{~kg}$ and $15.5 \mathrm{~kg}$, and only a few at $20 \mathrm{~kg}$, although some of them were able to produce maximal power at a weight of $25 \mathrm{~kg}$. In particular, water sports athletes performed best at higher weights.

The limitation of this study is that velocity and power during trunk rotations were not measured in a seated position in canoeists and kayakers, which would be more specific for these athletes. However, then it would not be possible to compare the velocity and power during trunk rotations with the remaining groups of athletes. Thus, the question remains as to whether they could perform better during seated trunk rotations. Standing rotational movement is less confined to the trunk and allows more involvement of the lower body. Therefore, power production is more effective during standing than seated trunk rotations [24]. When trunk rotations are performed in the sitting position, the involvement of the legs and the contribution of hips and the thoracic spine mobility, which allow the greatest rotation because of the orientation of the joints [32], to the upper-body rotational power, can be reduced. This could influence the performance in striking and throwing sports, in which the explosive strength and power are required for the production of movements in either the oblique or transverse planes [33]. The force is transferred from the proximal to the more distal segments. Because of the kinetic linkage between these segments [34-36], the rotational mobility may contribute to the force produced during rotations of the trunk. This force transfer can be crucial to throwing velocity. Therefore, exercises that closely replicate the rotation movements of the trunk should be preferred in functional testing of athletes in order to assess their sport-specific trunk rotational power.

\section{Conclusions}

Given that power and velocity produced during trunk rotations play an important role in athlete performance, their assessment should represent a crucial part of the testing battery. The findings showed significant between-group differences in mean power in the acceleration phase of trunk rotations, especially at higher weights $(\geq 10.5 \mathrm{~kg})$ or lower velocities $(\leq 334.2 \mathrm{rad} / \mathrm{s})$. The highest values of power were produced by fighting sports athletes with the maximum achieved at a lower weight of $10.5 \mathrm{~kg}$, followed by those of water sports at a higher weight of $20.0 \mathrm{~kg}$, combat sports at $15.5 \mathrm{~kg}$ and ball sports at $10.5 \mathrm{~kg}$. Similar tendencies were observed for angular velocity at different weights and force at different velocities. This indicates that these parameters are sensitive in revealing differences among athletes with different demands on the production of power and velocity under loading or unloading conditions, and could also reflect the specificity of their training involving trunk rotations.

This study is unique in assessing the sport-specific differences in muscle power and velocity during standing trunk rotations at different weights as well as power and force at different velocities in athletes of various sports. A better understanding of the role that power and velocity produced during trunk rotations play in athlete performance would help us to design specific exercise programs. Therefore, such testing should be implemented in sports that require the production of high force in a short time during trunk rotations. It may provide useful information on between- and within-group 
differences in muscle power produced during rotational exercises of the trunk and on the efficiency of their exercise programs.

Author Contributions: Conceptualization, E.Z., O.P. and M.J.; validation, E.Z., O.P. and M.J.; formal analysis, E.Z. and O.P.; data curation, E.Z., O.P. and M.J.; writing — original draft preparation, E.Z.; writing — review and editing, E.Z., O.P. and M.J.; project administration, E.Z. All authors have read and agreed to the published version of the manuscript.

Funding: This work was supported by the Scientific Grant Agency of the Ministry of Education, Science, Research and Sport of the Slovak Republic and the Slovak Academy of Sciences (Nos. 1/0824/17, 1/0089/20) and the Slovak Research and Development Agency under the contract No. APVV-15-0704.

Conflicts of Interest: The authors declare no conflict of interest.

\section{References}

1. Akuthota, V.; Nadler, S.F. Core strengthening. Arch. Phys. Med. Rehabil. 2004, 85, S86-S92. [CrossRef]

2. Martín-Moya, R.; Ruiz-Montero, P.J. Key aspects on physical fitness and injury prevention programs in football: A narrative review. J. Sport Health Res. 2017, 9, 311-328.

3. Barbado, D.; Barbado, L.C.; Elvira, J.L.L.; van Dieën, J.H.; Vera-Garcia, F.J. Sports-related testing protocols are required to reveal trunk stability adaptations in high-level athletes. Gait Posture 2016, 49, 90-96. [CrossRef]

4. Behm, D.G.; Drinkwater, E.J.; Willardson, J.M.; Cowley, P.M. The use of instability to train the core musculature. Appl. Physiol. Nutr. Metab. 2010, 35, 91-108. [CrossRef] [PubMed]

5. Keogh, J.W.L.; Aickin, S.E.; Oldham, A.R.H. Can common measures of core stability distinguish performance in a shoulder pressing task under stable and unstable conditions? J. Strength Cond. Res. 2010, 24, 422-429. [CrossRef] [PubMed]

6. Palmer, T.G.; Uhl, T.L. Interday reliability of peak muscular power outputs on an isotonic dynamometer and assessment of active trunk control using the chop and lift tests. J. Athl. Train. 2011, 46, 150-159. [CrossRef]

7. Andre, M.J.; Fry, A.C.; Heyrman, M.A.; Hudy, A.; Holt, B.; Roberts, C.; Vardiman, J.P.; Gallagher, P.M. A reliable method for assessing rotational power. J. Strength Cond. Res. 2012, 26, 720-724. [CrossRef] [PubMed]

8. Zemková, E.; Cepková, A.; Uvaček, M.; Šooš, L'. A novel method for assessing muscle power during the standing cable wood chop exercise. J. Strength Cond. Res. 2017, 31, 2246-2254. [CrossRef] [PubMed]

9. Talukdar, K.; Cronin, J.; Zois, J.; Sharp, A.P. The role of rotational mobility and power on throwing velocity. J. Strength Cond. Res. 2015, 29, 905-911. [CrossRef]

10. Nesser, T.W.; Huxel, K.C.; Tincher, J.L.; Okada, T. The relationship between core stability and performance in division I football players. J. Strength Cond. Res. 2008, 22, 1750-1754. [CrossRef]

11. Sato, K.; Mokha, M. Does core strength training influence running kinetics, lower-extremity stability, and 5000-M performance in runners? J. Strength Cond. Res. 2009, 23, 133-140. [CrossRef] [PubMed]

12. Scibek, J.S. The Effect of Core Stabilization Training on Functional Performance in Swimming. Ph.D. Thesis, University of North Carolina, Chapel Hill, NC, USA, 1999.

13. Stanton, R.; Reaburn, P.R.; Humphries, B. The effect of short-term Swiss ball training on core stability and running economy. J. Strength Cond. Res. 2004, 18, 522-528. [CrossRef] [PubMed]

14. Tse, M.A.; McManus, A.M.; Masters, R.S. Development and validation of a core endurance intervention program: Implications for performance in college-age rowers. J. Strength Cond. Res. 2005, 19, 547-552. [CrossRef] [PubMed]

15. Brown, E.W.; Abani, K. Kinematics and kinetics of the dead lift in adolescent power lifters. Med. Sci. Sports Exerc. 1985, 17, 554-566. [CrossRef] [PubMed]

16. Thelen, D.G.; Ashton-Miller, J.A.; Schultz, A.B. Lumbar muscle activities in rapid three-dimensional pulling tasks. Spine 1996, 21, 605-613. [CrossRef] [PubMed]

17. Stodden, D.F.; Fleisig, G.S.; McLean, S.P.; Lyman, S.L.; Andrews, J.R. Relationship of pelvis and upper torso kinematics to pitched baseball velocity. J. Appl. Biomech. 2001, 17, 164-172. [CrossRef]

18. Cholewicki, J.; VanVliet, J.J. 4th. Relative contribution of trunk muscles to the stability of the lumbar spine during isometric exertions. Clin. Biomech. 2002, 17, 99-105. [CrossRef]

19. Ellenbecker, T.S.; Roetert, E.P. An isokinetic profile of trunk rotation strength in elite tennis players. Med. Sci. Sports Exerc. 2004, 36, 1959-1963. [CrossRef] 
20. Abt, J.P.; Smoliga, J.M.; Brick, M.J.; Jolly, J.T.; Lephart, S.M.; Fu, F.H. Relationship between cycling mechanics and core stability. J. Strength Cond. Res. 2007, 21, 1300-1304. [CrossRef]

21. Aguinaldo, A.L.; Buttermore, J.; Chambers, H. Effects of upper trunk rotation on shoulder joint torque among baseball pitchers of various levels. J. Appl. Biomech. 2007, 23, 42-51. [CrossRef]

22. Lindsay, D.M.; Horton, J.F. Trunk rotation strength and endurance in healthy normals and elite male golfers with and without low back pain. N. Am. J. Sports Phys. Ther. 2006, 1, 80-88. [PubMed]

23. Suter, E.; Lindsay, D.M. Back muscle fatigability is associated with knee extensor inhibition in subjects with low back pain. Spine 2001, 26, E361-E366. [CrossRef] [PubMed]

24. Zemková, E.; Jeleň, M.; Zapletalová, L.; Hamar, D. Muscle power during standing and seated trunk rotations with different weights. Sport Mont. 2017, 15, 17-23. [CrossRef]

25. Zemková, E.; Jeleň, M.; Ollé, G.; Chren, M.; Olej, P.; Štefanovský, M.; Zapletalová, L.; Žiška, J.; Hamar, D. Mean power and velocity in acceleration phase of trunk rotation in athletes with different explosive force production capacity. In Proceedings of the 19th Annual Congress of the European College of Sport Science, Amsterdam, The Netherlands, 2-5 July 2014; p. 268.

26. Zemková, E.; Jeleň, M.; Ollé, G.; Hamar, D. Mean velocity of trunk rotation discriminates athletes with different sport-related demands. Eur. J. Sports Med. 2013, 1, 216.

27. Cohen, J. A power primer. Psychol. Bull. 1992, 112, 155-159. [CrossRef]

28. Shinkle, J.; Nesser, T.W.; Demchak, T.J.; McMannus, D.M. Effect of core strength on the measure of power in the extremities. J. Strength Cond. Res. 2012, 26, 373-380. [CrossRef]

29. Watkins, R.G.; Uppal, G.S.; Perry, J.; Pink, M.; Dinsay, J.M. Dynamic electromyographic analysis of trunk musculature in professional golfers. Eur. J. Appl. Physiol. Occup. Physiol. 1996, 24, 535-538. [CrossRef]

30. Poór, O.; Zemková, E. The effect of training in the preparatory and competitive periods on trunk rotational power in canoeists, ice-hockey players, and tennis players. Sports 2018, 6, 113. [CrossRef]

31. Tihanyi, J.; Apor, P.; Fekete, G. Force-velocity-power characteristics and fiber composition in human knee extensor muscles. Eur. J. Appl. Physiol. Occup. Physiol. 1982, 48, 331-343. [CrossRef]

32. Sahrmann, A.S. Diagnosis and Treatment of Movement Impairment Syndrome; Mosby Inc.: St Louis, MO, USA, 2002.

33. Earp, J.E.; Kraemer, W.J. Medicine ball training implications for rotational power sports. Strength Cond. J. 2010, 32, 20-25. [CrossRef]

34. Putnam, C.A. Sequential motions of body segments in striking and throwing skills: Descriptions and explanations. J. Biomech. 1993, 26, 125-135. [CrossRef]

35. Wagner, H.; Pfusterschmied, J.; Tilp, M.; Landlinger, J.; von Duvillard, S.P.; Müller, E. Upper-body kinematics in team-handball throw, tennis serve, and volleyball spike. Scand. J. Med. Sci. Sports 2014, 24, 345-354. [CrossRef] [PubMed]

36. Serrien, B.; Baeyens, J.-P. Systematic review and meta-analysis on proximal-to-distal sequencing in team handball: Prospects for talent detection? J. Hum. Kinet. 2018, 63, 9-21. [CrossRef] [PubMed]

Publisher's Note: MDPI stays neutral with regard to jurisdictional claims in published maps and institutional affiliations.

(C) 2020 by the authors. Licensee MDPI, Basel, Switzerland. This article is an open access article distributed under the terms and conditions of the Creative Commons Attribution (CC BY) license (http://creativecommons.org/licenses/by/4.0/). 